\title{
Ab initio simulations of the structure of thin water layers on defective anatase $\mathrm{TiO}_{2}(101)$ surfaces
}

\author{
Ulrich Aschauer ${ }^{1}$, Antonio Tilocca ${ }^{2}$, Annabella Selloni ${ }^{3, *}$ \\ 1. Materials Theory, ETH Zurich, 8093 Zürich, Switzerland. \\ 2. Department of Chemistry, University College London, London WC1H 0AJ, U.K. \\ 3. Department of Chemistry, Princeton University, Princeton, NJ 08544, USA.
}

\begin{abstract}
Titania-water interfaces are important in various fields of science, from geophysics to photocatalysis and biochemistry. Here we use ab initio molecular dynamics simulations to investigate the structure of thin water overlayers on the (101) surface of $\mathrm{TiO}_{2}$ anatase in the presence of oxidizing defects. For comparison, results of our previous studies of water layers on defect-free and reduced anatase (101) are also reviewed. On the stoichiometric defect-free surface ordered structures are formed at one and two monolayer coverage, and the order in the first bilayer is largely maintained when a third water layer is adsorbed. By contrast, the vertical and in-plane ordering of the water layers is strongly perturbed in the presence of both oxidizing and reducing defects. As a result, the structure of the water layer is much more diffuse, and frequent exchanges of water molecules between different layers are observed.
\end{abstract}




\section{1- Introduction}

The interaction of water with titania surfaces has attracted broad interest and stimulated intensive studies for decades. ${ }^{1,2}$ In particular, interest in the structure of adsorbed water layers on $\mathrm{TiO}_{2}$ is motivated by their relevance to essentially all applications of this material, ranging from photocatalysis and biocompatible devices to more traditional applications like pigments and coatings. ${ }^{3-6} \mathrm{TiO}_{2}$ has two main crystalline phases, rutile, which is the most stable polymorph of $\mathrm{TiO}_{2}$, and anatase, which is metastable but is predominant in nano-sized crystals. ${ }^{7,8}$ Anatase is also considered the $\mathrm{TiO}_{2}$ polymorph most efficient in photocatalysis ${ }^{9}$, which provides further motivation for the study of water adsorption on its surfaces.

Anatase (101) is the most abundant surface of anatase. ${ }^{8}$ It is strongly corrugated, with ridges of twofold coordinated bridging oxygen ions $\left(\mathrm{O}_{2 \mathrm{c}}\right)$ along the [010] direction (Figure 1a). ${ }^{10}$ It was shown theoretically ${ }^{11}$, and later confirmed by experiment ${ }^{12}$, that water adsorbs in molecular form on this surface, with the water oxygen $\left(\mathrm{O}_{\mathrm{w}}\right)$ binding to the unsaturated five-fold coordinated Ti cations $\left(\mathrm{Ti}_{5 \mathrm{c}}\right)$, while the water hydrogen atoms $\left(\mathrm{H}_{\mathrm{w}}\right)$ form $\mathrm{H}$-bonds with two $\mathrm{O}_{2 \mathrm{c}}$ atoms on the next ridge (Figure 1b). It was also theoretically predicted that surface oxygen vacancies ( $\mathrm{V}_{\mathrm{O}}$ 's) on anatase (101) would lead to water dissociation ${ }^{13}$, as observed in many studies of the rutile $\mathrm{TiO}_{2}(110)$ surface. $^{2}$ Unlike rutile (110), however, anatase (101) was experimentally found to have a very low concentration of surface $\mathrm{V}_{\mathrm{O}}$ ' $\mathrm{s}^{14,15}$ and in subsequent studies the predominance of subsurface oxygen vacancies was clearly established. ${ }^{16,17}$

The influence of subsurface $\mathrm{V}_{\mathrm{O}}$ 's and $\mathrm{Ti}$ interstitials (another typical $\mathrm{TiO}_{2}$ defect) on water adsorption in the dilute limit was investigated by Aschauer et al. ${ }^{18}$. The calculations showed a preference for water to adsorb in the vicinity of the subsurface defects, as well as a stronger tendency for water to dissociate on the defected surface than on the defect-free one. For higher water coverages, the structure and reactivity of thin water layers on defect-free and defective anatase (101) surfaces were investigated by Tilocca and Selloni using ab initio molecular dynamics (AIMD) simulations. ${ }^{19,20}$ Compared to the defect-free surface, subsurface defects were found to enhance the surface reactivity and to lead to a more disordered structure of the first water layers adsorbed on the reduced surface. In particular, no water dissociation took place in rather long simulations for a water monolayer, bilayer and trilayer on the defect-free surface, whereas dissociated water was observed on the defected surface. 
While oxygen vacancies and $\mathrm{Ti}$ interstitials $\left(\mathrm{Ti}_{\text {int }}\right.$ 's) are almost invariably present in $\mathrm{TiO}_{2}$, causing the material to be reduced and conductive (both $\mathrm{V}_{\mathrm{O}}$ 's and $\mathrm{Ti}_{\text {int }}$ 's are electron donors) ${ }^{7}$, recently oxidizing defects with the character of "bridging oxygen dimers" have been reported to exist on the anatase (101) surface. ${ }^{21}$ It was also suggested that these defects - denoted $\left(\mathrm{O}_{2}\right)_{\mathrm{O}}$ 's play an important role in water oxidation, which makes their study particularly interesting. ${ }^{22}$ The main purpose of this work is thus to investigate how the oxidizing $\left(\mathrm{O}_{2}\right)_{\mathrm{O}}$ defects affect the structure of adsorbed water layers on the anatase (101) surface by AIMD simulations analogous

to those we previously reported for the stoichiometric and reduced surfaces. ${ }^{19,20}$ Another aim is to compare the present results for oxidized (i.e. electron-poor) anatase (101) to our previous studies for the stoichiometric and reduced (i.e. electron-rich) surfaces in order to explore similarities and differences between different types of defects as well as possible correlations between the surface electronic structure and the structure of adsorbed water. To make the present work more self-contained, a review of our previous studies on the stoichiometric and reduced anatase surfaces is included in the following.

\section{2- Computational methods}

All calculations of this work were performed using Density Functional Theory within the Generalized Gradient Approximation (GGA) of Perdew-Burke-Ernzerhof ${ }^{23}$ (PBE) approximation as implemented in the Quantum ESPRESSO package. ${ }^{24}$ The adequacy of this approximation for discussing the structure of adsorbed water on the reduced anatase (101) surface has been tested and discussed in detail in Ref. ${ }^{20}$. It was shown there that the DFT+U and DFT-GGA descriptions of the structural and dynamical features of a water monolayer on the reduced surface are sufficiently close to justify the use of the standard DFT-GGA approach to investigate the structure of thin water films. Electron-ion interactions were described using ultrasoft pseudopotentials, ${ }^{25}$ with $\mathrm{Ti}(3 \mathrm{~s}, 3 \mathrm{p}, 3 \mathrm{~d}, 4 \mathrm{~s}), \mathrm{O}(2 \mathrm{~s}, 2 \mathrm{p})$ and $\mathrm{H}(1 \mathrm{~s})$ electrons treated explicitly as valence electrons. Wavefunctions were expanded in plane waves up to a kinetic energy cutoff of $25 \mathrm{Ry}$, while a cutoff of 200 Ry was used for the augmented density.

The anatase (101) surface was modeled as a periodically repeated slab of three $\mathrm{TiO}_{2}$ layers, with vacuum regions of $10 \AA$ separating consecutive water-covered slabs along the surface normal. Water molecules were adsorbed on one side of the slab only. Different water coverages were considered, from submonolayer up to three monolayers, where one monolayer (ML) corresponds 
to one molecule per Ti5c site. For static calculations we used a $1 \times 3$ surface supercell of dimensions $10.262 \AA \times 11.310 \AA$ exposing six $\mathrm{Ti}_{5 \mathrm{c}}$ sites, while for molecular dynamics a $1 \times 4$ surface supercell of dimensions $10.262 \AA$ x $15.080 \AA$ exposing eight $\mathrm{Ti}_{5 \mathrm{c}}$ sites was considered. The atoms at the bottom of the slab were kept fixed in all cases. Reciprocal space sampling was restricted to the $\Gamma$-point.

Structural relaxations were carried out until forces converged below $0.05 \mathrm{eV} / \AA$ and reaction barriers were determined using the climbing-image nudged-elastic band ${ }^{26}$ (NEB) method. Molecular dynamics calculations were carried out using the Car-Parrinello ${ }^{27}$ method with a timestep of 5 a.u., a fictitious electron mass of 500 a.u. and deuterium masses for the hydrogen atoms. The temperature was kept at $160 \mathrm{~K}$ using a Nosé-Hoover thermostat with an oscillation frequency of $20 \mathrm{THz}$. All configurations have been equilibrated for 1.5 ps prior to recoding data for $20 \mathrm{ps}$.

\section{3- Review of previous results}

\subsection{Thin water layers on stoichiometric anatase (101)}

The structures of one (ML), two (BL), and three (TL) water layers on the defect-free surface were investigated by AIMD simulations at $\mathrm{T}=160 \mathrm{~K}$, the temperature at which desorption of adsorbed water is observed to start in temperature programmed desorption (TPD) experiments. Snapshots from these simulations (Figure 2a) suggest the presence of a partially layered structure. A more detailed analysis of this structure is reported in Figure 3a, which shows the time evolution of the vertical distances of the water molecules from the surface along with their probability distribution. We can see that the structures of the ML and BL are characterized by all molecules being at well-defined distances from the surface and corresponding sharp peaks in the distance distributions at $\Delta \mathrm{z}=2$ and $3 \AA$. The left and middle panels in Figure 2a show that water molecules in the first layer $(\Delta \mathrm{z}=2 \AA)$ are coordinated to $\mathrm{Ti}_{5 \mathrm{c}}$, whereas the second layer $(\Delta \mathrm{z}=$ $3 \AA$ ) is formed by strongly oriented molecules, all arranged with one hydrogen pointing upward and the other toward the surface and forming a strong $\mathrm{H}$-bond with an $\mathrm{O}_{2 \mathrm{c}}$. The vertical ordering of the first two layers in contact with the surface is maintained also in the TL, but the presence of the additional water molecules introduces some disorder. The peak corresponding to the third layer in the height distribution is at $\Delta \mathrm{z} \sim 4.5 \AA$ but has a tail extending up to $6 \AA$, suggesting a higher mobility and a more disordered arrangement within the top layer. 
The pair correlation functions are shown in Figure 4a, where the upper and lower panels refer to the water-water and water-surface interactions, respectively. In the upper panel, we can see that $\mathrm{Ti}_{5_{c}}$-coordinated water molecules in the ML do not significantly interact with each other, due to the large $\mathrm{Ti}_{5_{\mathrm{c}}}-\mathrm{Ti}_{5_{\mathrm{c}}}$ distances. Clear signatures of a H-bonded system (intermolecular $\mathrm{O}_{\mathrm{w}}-\mathrm{H}_{\mathrm{w}}$ and $\mathrm{O}_{\mathrm{w}}-\mathrm{O}_{\mathrm{w}}$ peaks at 1.7 and $2.7 \AA$, respectively) emerge for the $\mathrm{BL}$, and are maintained for the TL, with a generally more disordered arrangement evident from the broader peaks. In the lower panel, the persistent $\mathrm{Ti}-\mathrm{O}_{\mathrm{w}}$ peak around $2.3 \AA$ indicates that water molecules remain strongly coordinated to $\mathrm{Ti}_{5 \mathrm{c}}$ at the different coverages. Water molecules in the ML are anchored to the $\mathrm{Ti}_{5 \mathrm{c}}$ sites and can only form weak $\mathrm{H}$-bonds with the surface $\mathrm{O}_{2 \mathrm{c}}$. On the other hand, the $\mathrm{BL}$ and $\mathrm{TL}$ also include molecules that are not coordinated to $\mathrm{Ti}_{5 \mathrm{c}}$ and are thus able to form stronger $\mathrm{H}-$ bonds with the surface. In particular, water-surface H-bonds are strongest in the BL due to the contribution from strongly oriented molecules pointing a hydrogen downwards (Figure 2a), which leads to sharp $\mathrm{H}_{\mathrm{w}}-\mathrm{Os}$ and $\mathrm{Ow}-\mathrm{Os}$ peaks at 1.5 and $2.5 \AA$.

\subsection{Thin water layers on reduced anatase (101)}

The reduced surface was modeled by adding a Ti interstitial atom in the second layer, see Figure 2b. Similar to the oxygen vacancy, this defect is an electron donor and gives rise to $\mathrm{Ti}^{3+}$ species, which are a characteristic signature of reduced $\mathrm{TiO}_{2}$. While hybrid functionals or DFT+U methods are generally required to obtain a satisfactory description of the electronic properties of $\mathrm{Ti}^{3+}$ species, ${ }^{28}$ DFT-GGA and DFT+U were found to predict similar energetic and dissociation barrier for an isolated adsorbed water molecule, as well as very similar structural features for an adsorbed water monolayer on the reduced surface, thus justifying the use of standard DFT-GGA for the simulation of thicker water layers. ${ }^{20}$

Snapshots from AIMD simulations of 1-3 water layers on the reduced surface at $160 \mathrm{~K}$ are shown in Figure $2 b$. We can notice that the presence of the interstitial substantially affects the ML structure compared to the defect-free surface. The ordered arrangement of $\mathrm{Ti}_{5 \mathrm{c}}$-coordinated molecules observed on the stoichiometric surface changes into a partially dissociated layer with an empty $\mathrm{Ti}_{5 \mathrm{c}}$ site, due to the migration of the one water molecule to an intermediate position between the $\mathrm{Ti}_{5 \mathrm{c}}$ and $\mathrm{O}_{2 \mathrm{c}}$ rows. The subsurface defect has an even more marked influence on the BL structure, whose vertical arrangement changes from the ordered double layer with sharp peaks seen on the defect-free surface to a multi-peak distribution reaching up to $8 \AA$ from the 
surface (Figure 3b). Even though the peaks at 2 and $3 \AA$ characteristic of the BL on the defectfree surface are still present, the structure of these layers is significantly perturbed (Figure $2 b$ ). For the TL, the structure of the first two layers in contact with the surface is similar to that just described for the BL, with a dissociated water in the first layer and a depleted second layer of molecules H-bonded to the surface. Above these two layers in direct contact with the surface sites, the remaining molecules are significantly spread for about $3 \AA$ perpendicularly to the surface.

The radial distribution functions for water on the defective surface (Figure $4 \mathrm{~b}$ ) provide further evidence of the substantial influence of the interstitial defect on the structure of adsorbed water. At variance with the defect-free surface, signatures of hydrogen bonds between water molecules are observed already in the ML. In this case, H-bonds always involve $\mathrm{OH}$ groups produced by water dissociation and are stronger than $\mathrm{H}$-bonds between undissociated water molecules, which become dominant in the bi- and trilayer: $\mathrm{O}_{\mathrm{w}}-\mathrm{O}_{\mathrm{w}}$ and $\mathrm{O}_{\mathrm{w}}-\mathrm{H}_{\mathrm{w}}$ peaks lie at 2.5 and $1.45 \AA$ in the ML, compared to values of 2.7 and $1.7 \AA$ in the $\mathrm{BL}$ and TL. The water-water hydrogen bond patterns in the $\mathrm{BL}$ and TL are very similar, with distances approaching those of a liquid water environment. The water-surface pair correlation functions are shown in the bottom panel of Figure $4 \mathrm{~b}$. The formation of a $\mathrm{Ti}-\mathrm{OH}$ bond results in the short-distance peak in the $\mathrm{Ti}^{-}-\mathrm{O}_{\mathrm{w}}$ radial distribution function. This feature is most prominent for the $\mathrm{ML}$ and increasingly less for the BL and TL. At variance with the defect-free surface, hydrogen bonds between water and the reduced surface are already present at ML coverage.

\section{4 - Water on oxidized anatase (101)}

\subsection{Adsorption energies at low coverage}

As mentioned in the introduction, the anatase (101) surface can assume an oxidized state by accommodating an $\mathrm{O}_{2}$ molecule at the lattice position of a bridging $\mathrm{O}_{2 \mathrm{c}}$ oxygen. The structure of the resulting $\left(\mathrm{O}_{2}\right)_{\mathrm{O}}$ bridging dimer defect is shown in Figure 5. In this figure the inequivalent $\mathrm{Ti}_{5 \mathrm{c}}$ adsorption sites around the $\left(\mathrm{O}_{2}\right)_{\mathrm{O}}$ defect are labeled from 1 to 6 ; note that site 2 and 3 as well as 4 and 6 are equivalent by symmetry. The computed adsorption energies for a water molecule at these different $\mathrm{Ti}_{5_{\mathrm{c}}}$ surface sites are given in Table 1, where the adsorption energy $(-0.71 \mathrm{eV})$ on the stoichiometric defect-free surface is also reported as a reference. We can see that the bridging dimer has almost no effect on adsorption at sites 1,2 and 4, which are further away 
from the dimer than site 5, the closest to the defect. At this latter site we compute a slight enhancement of the adsorption energy by $0.11 \mathrm{eV}$ compared to the stoichiometric surface. The $\mathrm{Ti}_{5 \mathrm{c}}-\mathrm{O}_{\mathrm{w}}$ bond length at this site is slightly shorter than for the other sites, reflecting the stronger bond. The H-bond lengths are generally longer than on the stoichiometric surface, the lengthening being small at sites 1 and 5, where the structural changes due to the $\left(\mathrm{O}_{2}\right)_{\mathrm{O}}$ are small. For site 2 a very asymmetric bonding pattern is observed, in which the H-bond not leading to the $\left(\mathrm{O}_{2}\right)_{\mathrm{O}}$ is significantly longer, whereas the bond to the $\left(\mathrm{O}_{2}\right)_{\mathrm{O}}$ is shorter than the one on the stoichiometric surface. We would expect site 4 to show a rather unaffected bonding pattern but see significantly longer H-bond lengths, which cannot be explained by surface geometry changes but must be due to a reduction of the H-bond strength at this site.

Table I: Adsorption energies for a water molecule at various inequivalent $\mathrm{Ti}_{5 \mathrm{c}}$ sites (Figure 5) on the oxidized anatase (101) surface compared to the adsorption energy on the stoichiometric surface. The $\mathrm{Ti}_{\mathrm{w}} \mathrm{O}_{\mathrm{w}}$ and $\mathrm{O}-\mathrm{H}_{\mathrm{w}}$ bond lengths are given as well.

\begin{tabular}{l|l|l|l|l} 
Site & $\begin{array}{l}\text { Adsorption } \\
\text { energy (eV) }\end{array}$ & $\begin{array}{l}\mathbf{T i}_{5 c}-\mathbf{O}_{\mathbf{w}} \\
(\mathbf{\AA})\end{array}$ & $\begin{array}{l}\mathbf{O}_{2 \mathbf{c}}-\mathbf{H}_{\mathbf{w 1}} \\
(\mathbf{\AA})\end{array}$ & $\begin{array}{l}\mathbf{O}_{2 \mathbf{c}}-\mathbf{H}_{\mathbf{w} \mathbf{2}} \\
(\mathbf{\AA})\end{array}$ \\
\hline 1 & -0.72 & 2.29 & 2.27 & 2.30 \\
$2=3$ & -0.68 & 2.28 & 2.50 & 2.24 \\
$4=6$ & -0.70 & 2.29 & 2.37 & 2.40 \\
5 & -0.82 & 2.27 & 2.28 & 2.28 \\
\hline Stoichiometric & -0.71 & 2.30 & 2.26 & 2.26
\end{tabular}

The water adsorption energy and dissociation barrier for a water molecule at site 5 on oxidized anatase (101) are compared to analogous NEB calculations for the stoichiometric and reduced surfaces in Figure 6. Both reduced and oxidized anatase (101) bind molecular water more strongly than the stoichiometric surface. For the oxidized surface, the molecular adsorption energy increases to $0.82 \mathrm{eV}$ compared to $0.71 \mathrm{eV}$ on the stoichiometric surface. For the reduced surface, the adsorption energy is 1.02 and $1.04 \mathrm{eV}$ when the reduction is caused by an oxygen vacancy ( 2 electron donor) and a Ti interstitial (4 electron donor), respectively. The effect of surface reduction or oxidation on the adsorption energy of the dissociated state is similar. On the stoichiometric surface we compute the least favorable dissociated adsorption energy of $0.31 \mathrm{eV}$, i.e. dissociation is endothermic by $0.40 \mathrm{eV}$. On the oxidized surface the adsorption strength of the dissociated state increases to $0.58 \mathrm{eV}$, so that dissociation is endothermic by $0.25 \mathrm{eV}$. 
Reducing the surface with an oxygen vacancy brings the molecular and dissociated states even closer in energy, so that dissociation is now endothermic by only $0.13 \mathrm{eV}$. The Ti interstitial finally renders dissociation slightly exothermic by $0.03 \mathrm{eV}$. Looking at the barriers for dissociation, we can see that the highest barrier is obtained for the stoichiometric surface $(0.53$ $\mathrm{eV})$, followed by the oxidized surface $(0.48 \mathrm{eV})$. The bridging dimer thus has a fairly shortrange effect on water adsorption and slightly affects dissociation energetics and kinetics. Significantly smaller dissociation barriers are found for the reduced surfaces, 0.26 and $0.24 \mathrm{eV}$ for the $\mathrm{O}$ vacancy and the Ti interstitial respectively.

\subsection{Thin water layers on oxidized anatase (101)}

As in our previous studies for stoichiometric and reduced anatase (101), the dynamic structure of a monolayer (ML), bilayer (BL), and trilayer (TL) of water on the oxidized surface was investigated by AIMD simulations at $\mathrm{T}=160 \mathrm{~K}$. In the left hand side of Figure 7 we show the time evolution of the vertical distance of the water molecule's center of mass with respect to the instantaneous average of the $\mathrm{Ti}_{5 \mathrm{c}}$ surface sites. From Figure $7 \mathrm{a}$, we can see that one water molecule is expelled from the ML just after the equilibration period. The missing water molecule away from the dimer (at a position that would correspond to site 1 in Figure 5) is clearly visible in the snapshot in Figure 7g. After $\sim 12 \mathrm{ps}$ the molecule returns into the ML, but this gives rise to the almost immediate expulsion of another water molecule followed by a second one about 2ps later. By comparing the positions of these expulsed molecules to those of the bilayer in Figure 7b, we can see that their position indeed coincides with the second layer of water above the surface. Similar to the ML, in the BL case we can see two water molecules being expelled from the first layer at around 2ps (Figure 7b). They then assume a position slightly above the second layer but well below the third layer, which is populated by a single water molecule expelled from the second layer already during equilibration. In the snapshot in Figure $7 \mathrm{~h}$, the water molecule in the first layer is missing at the very left (site 6). For the TL case, the water molecules just above the second layer disappear, forming instead a more diffuse layer around $5 \AA$ above the $\mathrm{Ti}_{5 \mathrm{c}}$. In this situation we can also observe a few water exchanges between the first and second layer as well as the second and higher layers. The probability distribution in Figure f show well-defined peaks for the first and second layer as well as a more diffuse third layer, a structure that is also evident in the snapshot in Figure $7 \mathrm{i}$. 
The expulsion of water molecules from the regular layered structure that we here observe for the oxidized surface does not occur on the stoichiometric surface (Figure 3a), but is present also on the reduced surface (Figure $3 b$ ). In the present case it is likely the result of a disturbance in H-bonding. Adsorbed water molecules to the left of the bridging dimer (at sites 2 and 3 in Figure 5) form $\mathrm{H}$-bonds with the dimer. The latter is in a peroxide $\mathrm{O}_{2}{ }^{2-}$ state and thus provides less charge per $\mathrm{O}$ atom for bonding than a regular surface $\mathrm{O}^{2-}$ ion. Moreover the $\mathrm{H}$-bond distances are significantly shorter than for regular sites thus imposing a different local geometry. This leads to a reduced water adsorption energy at these sites as shown in Table I. On the other hand, the stronger binding energy of the water molecule at site 5 also causes a disturbance of the water H-bond network which favors desorption and expulsion of water at sites further away from the dimer.

In order further quantify the local structure of the adsorbed layers, the correlation functions for various relevant pairs are shown in Figure 8. By comparing the water-water pair correlation functions for ML, BL and TL in the first column, it appears that the peak positions of the water structure do not change significantly for layers of increasing thickness even though the height of the peaks changes as the fraction of corresponding distances varies. In the second column, which reports the $\mathrm{O}_{\mathrm{w}}$-Ti correlations, the position of the first peak remains the same for all thicknesses of the water overlayer. The positions of the subsequent peaks show the same tendency observed in the water layer positions in Figures $7 \mathrm{~d}$-f. While the bonding of water with the $\mathrm{Ti}_{5 \mathrm{c}}$ sites seems not much affected by the dimer, we can see interesting differences in the pair distribution functions especially for the $\mathrm{H}_{\mathrm{w}}-\mathrm{O}_{\mathrm{s}}$ distances in the fourth column of Figure 8. In all these plots we see a shift to slightly larger distances for the first (H-bonding) $\mathrm{H}_{\mathrm{w}}-\mathrm{O}_{\text {dimer }}$ peak, which is in agreement with the decreased H-bonding strength around the dimer. Comparing the two curves for $\mathrm{H}_{\mathrm{w}}-\mathrm{O}_{2 \mathrm{c}}$ and $\mathrm{H}_{\mathrm{w}}-\mathrm{O}_{\text {dimer }}$ pairs in Figure $8 \mathrm{~d}$ we can further see that the dimer induces a different local structure with additional peaks, which is likely at the origin of the dynamical effects in Figure 7. This disturbance is also visible for the BL (Figure 8h) and to a lesser extent in for the TL (Figure 81). Similar shifts of the first peak are also observed in the $\mathrm{O}_{\mathrm{w}}-\mathrm{O}_{\mathrm{s}}$ correlations in the third column of Figure 8, together with the appearance of additional peaks due to the dimer, in regions where normally no peaks are found. This demonstrates how the bridging dimer locally imposes a different water structure in comparison to that on the stoichiometric surface, thus causing a disruption of the regular water structure in the vicinity of the dimer. 


\section{Conclusions}

In this work we have studied the structure of thin water overlayers on defect-free and defected (101) surfaces of $\mathrm{TiO}_{2}$ anatase by ab initio molecular dynamics simulations. On the stoichiometric anatase(101) surface, water-surface interactions dominate over intermolecular water-water interactions. This leads to substantial vertical and in-plane ordering of the first two layers in direct contact with the surface, which is maintained even at higher coverages, although surface-induced ordering is weaker above the second layer. By contrast, both reducing and oxidizing defects, notably subsurface titanium interstitials (Figure $3 b$ ) and surface bridging oxygen dimers (Figure 7), lead to a disruption of the regular water structure observed on the stoichiometric surface (Figure 3a). We note however that the mechanisms for this disruption are different on the reduced and oxidized surfaces. On the oxidized surface H-bonds formed with the $\left(\mathrm{O}_{2}\right)_{\mathrm{O}}$ dimer rather than a regular $\mathrm{O}_{2 \mathrm{c}}$ surface site lead to a locally altered H-bonding pattern around the dimer. This perturbation of the regular H-bond structure results in desorption of water molecules from $\mathrm{Ti}_{5 \mathrm{c}}$ sites and their migration to subsequent water layers where they replace other water molecules. On the reduced surface we also observe desorption of a water molecule and its migration to higher lying layers. In contrast to the oxidized surface, the process on the reduced surface is however triggered by water dissociation and a stronger H-bond formation between water and the resulting $\mathrm{OH}$ groups. This leads to a more marked and long-range disturbance of the water structure above the reduced surface as seen by the diffuse peaks in Figure 3b compared to the almost unaltered peak structure for the oxidized surface shown in Figure 7. On the basis of these results we can also expect a difference in the dynamical properties of the interfacial water on the oxidized and reduced surfaces, with higher water diffusivity on the latter. Our study thus reveals how small details of the surface structure can critically affect the properties of the adsorbed water layers at the interface.

\section{Acknowledgement}

This work was supported by DoE-BES, Division of Chemical Sciences, Geosciences and Biosciences under Award DE-FG02-12ER16286. We used resources of the National Energy 
Research Scientific Computing Center (DoE Contract No. DE-AC02-05CH11231). We also acknowledge use of the TIGRESS high performance computer center at Princeton University.

\section{REFERENCES}

1 Henderson, M. A. The interaction of water with solid surfaces: fundamental aspects revisited. Surf. Sci. Rep. 46, 1 - 308 (2002).

2 Sun, C. H., Liu, L. M., Selloni, A., Lu, G. Q. \& Smith, S. C. Titania-water interactions: a review of theoretical studies. J. Mater. Chem. 20, 10319-10334, doi:10.1039/c0jm01491e (2010).

3 Linsebigler, A. L., Lu, G. \& Yates, J. T., Jr. Photocatalysis on TiO2. Chem. Rev. 95, 735-758 (1995).

4 Fujishima, A., Zhang, X. T. \& Tryk, D. A. TiO2 photocatalysis and related surface phenomena. Surf. Sci. Rep. 63, 515-582 (2008).

5 Henderson, M. A. A surface science perspective on TiO2 photocatalysis. Surf. Sci. Rep. 66, 185297, doi:10.1016/j.surfrep.2011.01.001 (2011).

6 Chen, X. \& Selloni, A. Introduction: Titanium Dioxide (TiO2) Nanomaterials. Chemical Reviews 114, 9281-9282, doi:10.1021/cr500422r (2014).

7 Diebold, U. The surface science of titanium dioxide. Surf. Sci. Rep. 48, 53-229 (2003).

8 Diebold, U., Ruzycki, N., Herman, G. S. \& Selloni, A. One step towards bridging the materials gap: surface studies of TiO2 anatase. Catalysis Today 85, 93-100 (2003).

9 Kavan, L., Grätzel, M., Gilbert, S. E., Klemenz, C. \& Scheel, H. J. Electrochemical and Photoelectrochemical Investigation of Single-Crystal Anatase. J. Amer. Chem. Soc. 118, 67166723, doi:10.1021/ja954172l (1996).

10 Lazzeri, M., Vittadini, A. \& Selloni, A. Structure and energetics of stoichiometric TiO2 anatase surfaces. Phys. Rev. B 63, 155409/155401-155409/155409 (2001).

11 Vittadini, A., Selloni, A., Rotzinger, F. P. \& Gratzel, M. Structure and Energetics of Water Adsorbed at TiO2 Anatase (101) and (001) Surfaces. Phys. Rev. Lett. 81, 2954-2957 (1998).

12 He, Y. B., Tilocca, A., Dulub, O., Selloni, A. \& Diebold, U. Local ordering and electronic signatures of submonolayer water on anatase TiO2(101). Nature Materials 8, 585-589 (2009).

13 Tilocca, A. \& Selloni, A. Reaction pathway and free energy barrier for defect-induced water dissociation on the (101) surface of TiO2-anatase. Journal of Chemical Physics 119, 7445-7450, doi:10.1063/1.1607306 (2003).

14 Hebenstreit, W., Ruzycki, N., Herman, G. S., Gao, Y. \& Diebold, U. Scanning tunneling microscopy investigation of the TiO2 anatase (101) surface. Phys. Rev. B: Condensed Matter and Materials Physics 62, R16334-R16336 (2000).

15 Thomas, A. G. et al. Comparison of the electronic structure of anatase and rutile TiO[sub 2] single-crystal surfaces using resonant photoemission and x-ray absorption spectroscopy. Phys. Rev. B (Condensed Matter and Materials Physics) 75, 035105 (2007).

16 Cheng, H. \& Selloni, A. Surface and subsurface oxygen vacancies in anatase TiO[sub 2] and differences with rutile. Phys. Rev. B (Condensed Matter and Materials Physics) 79, 092101 (2009).

17 He, Y., Dulub, O., Cheng, H., Selloni, A. \& Diebold, U. Evidence for the Predominance of Subsurface Defects on Reduced Anatase TiO[sub 2](101). Phys. Rev. Lett. 102, 106105 (2009).

18 Aschauer, U. et al. Influence of Subsurface Defects on the Surface Reactivity of TiO2: Water on Anatase (101). J. Phys. Chem. C 114, 1278 (2010). 
Tilocca, A. \& Selloni, A. Vertical and lateral order in adsorbed water layers on anatase TiO2(101). Langmuir 20, 8379-8384, doi:10.1021/la048937r (2004).

20 Tilocca, A. \& Selloni, A. DFT-GGA and DFT+U Simulations of Thin Water Layers on Reduced TiO2 Anatase. J. Phys. Chem. C 116, 9114-9121, doi:10.1021/jp301624v (2012).

21 Setvin, M. et al. Reaction of $\mathrm{O} 2$ with subsurface oxygen vacancies on TiO2 anatase (101). Science 341, 988-991, doi:10.1126/science.1239879 (2013).

22 Li, Y.-F., Liu, Z.-P., Liu, L. \& Gao, W. Mechanism and Activity of Photocatalytic Oxygen Evolution on Titania Anatase in Aqueous Surroundings. J. Amer. Chem. Soc. 132, 13008-13015, doi:10.1021/ja105340b (2010).

23 Perdew, J. P., Burke, K. \& Ernzerhof, M. Generalized gradient approximation made simple. Physical Review Letters 77, 3865-3868, doi:10.1103/PhysRevLett.77.3865 (1996).

24 Quantum ESPRESSO (http://www.democritos.it).

25 Vanderbilt, D. Soft Self-Consistent Pseudopotentials In A Generalized Eigenvalue Formalism. Physical Review B 41, 7892-7895 (1990).

26 Henkelman, G., Uberuaga, B. P. \& Jónsson, H. A climbing image nudged elastic band method for finding saddle points and minimum energy paths. Journal of Chemical Physics 113, 9901-9904 (2000).

27 Car, R. \& Parrinello, M. Unified approach for molecular dynamics and density-functional theory. Phys. Rev. Lett. 55, 2471 (1985).

28 De Angelis, F., Di Valentin, C., Fantacci, S., Vittadini, A. \& Selloni, A. Theoretical Studies on Anatase and Less Common TiO2 Phases: Bulk, Surfaces, and Nanomaterials. Chemical Reviews 114, 9708-9753, doi:10.1021/cr500055q (2014). 


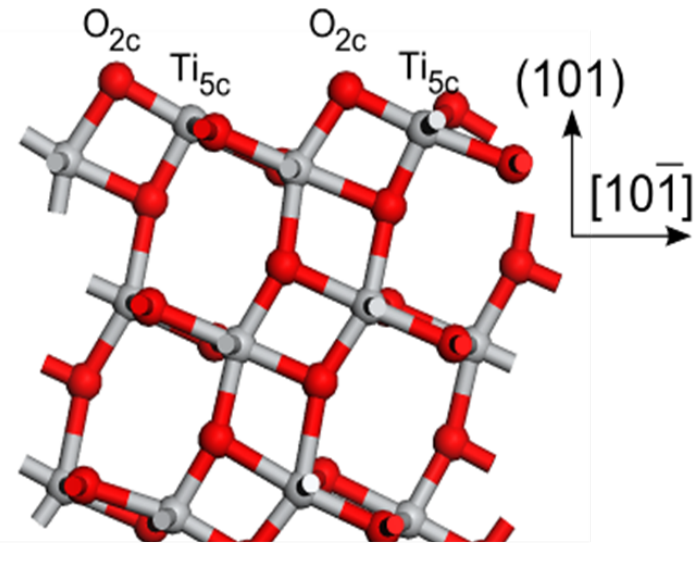

(a)

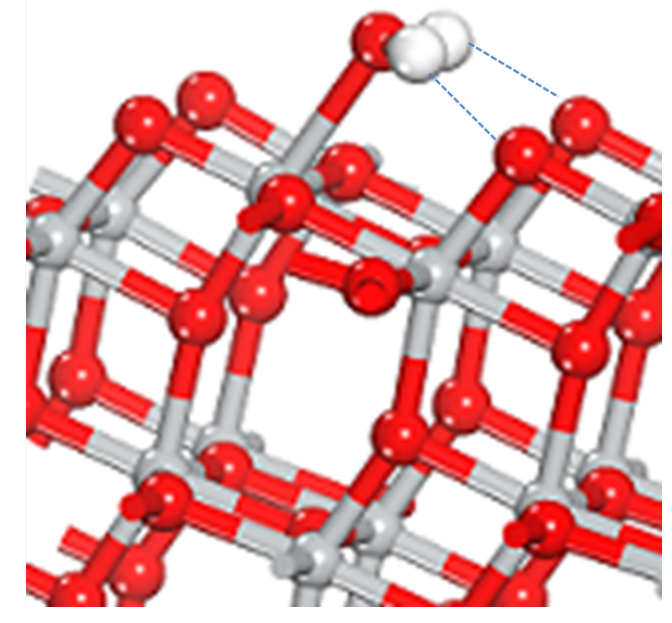

(b)

Figure 1 - Structure of: (a) the bare anatase (101) surface (side view), and (b) the same surface with an adsorbed water molecule (perspective view). In (a), the undercoordinated $\mathrm{O}_{2 \mathrm{c}}$ and $\mathrm{Ti}_{5 \mathrm{c}}$ surface sites are indicated. The dashed lines in (b) highlight the H-bonds formed by the adsorbed molecule with the $\mathrm{O}_{2 \mathrm{c}}$ ions. $\mathrm{Ti}$ and $\mathrm{O}$ atoms are shown in gray and red, respectively. 


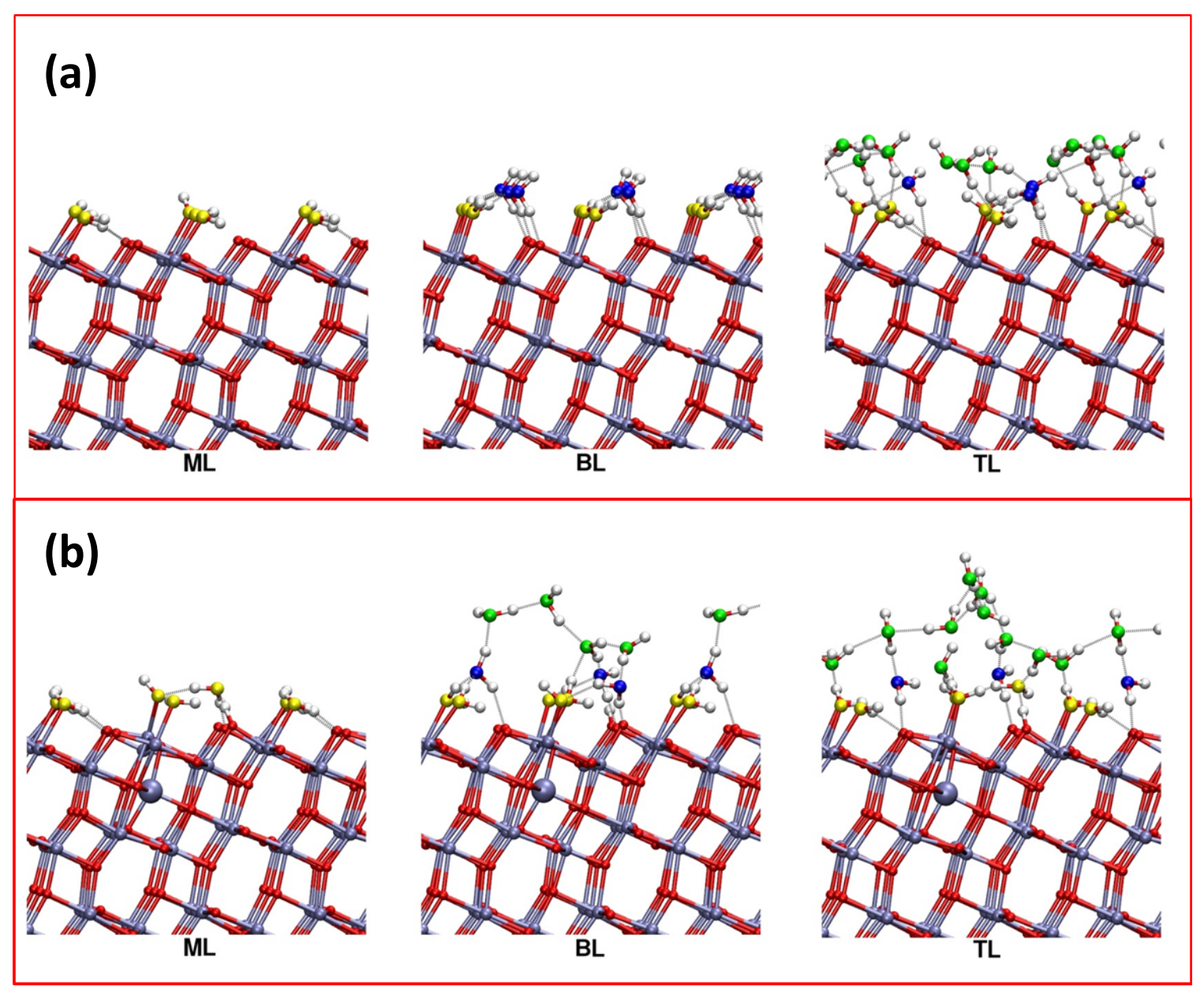

Figure 2. Snapshots of a mono-, bi-, and trilayer of water on the defect-free (a) and reduced (b) anatase (101) surfaces, from AIMD simulations at $160 \mathrm{~K}$. Note the presence of a dissociated water molecule in (b). Water molecules bonded to undercoordinated $\mathrm{Ti}_{5 c}$ and bridging $\mathrm{O}_{2 c}$ sites are highlighted in yellow and blue, respectively, whereas the remaining molecules are green. The large gray sphere in (b) represents the Ti interstitial defect. 

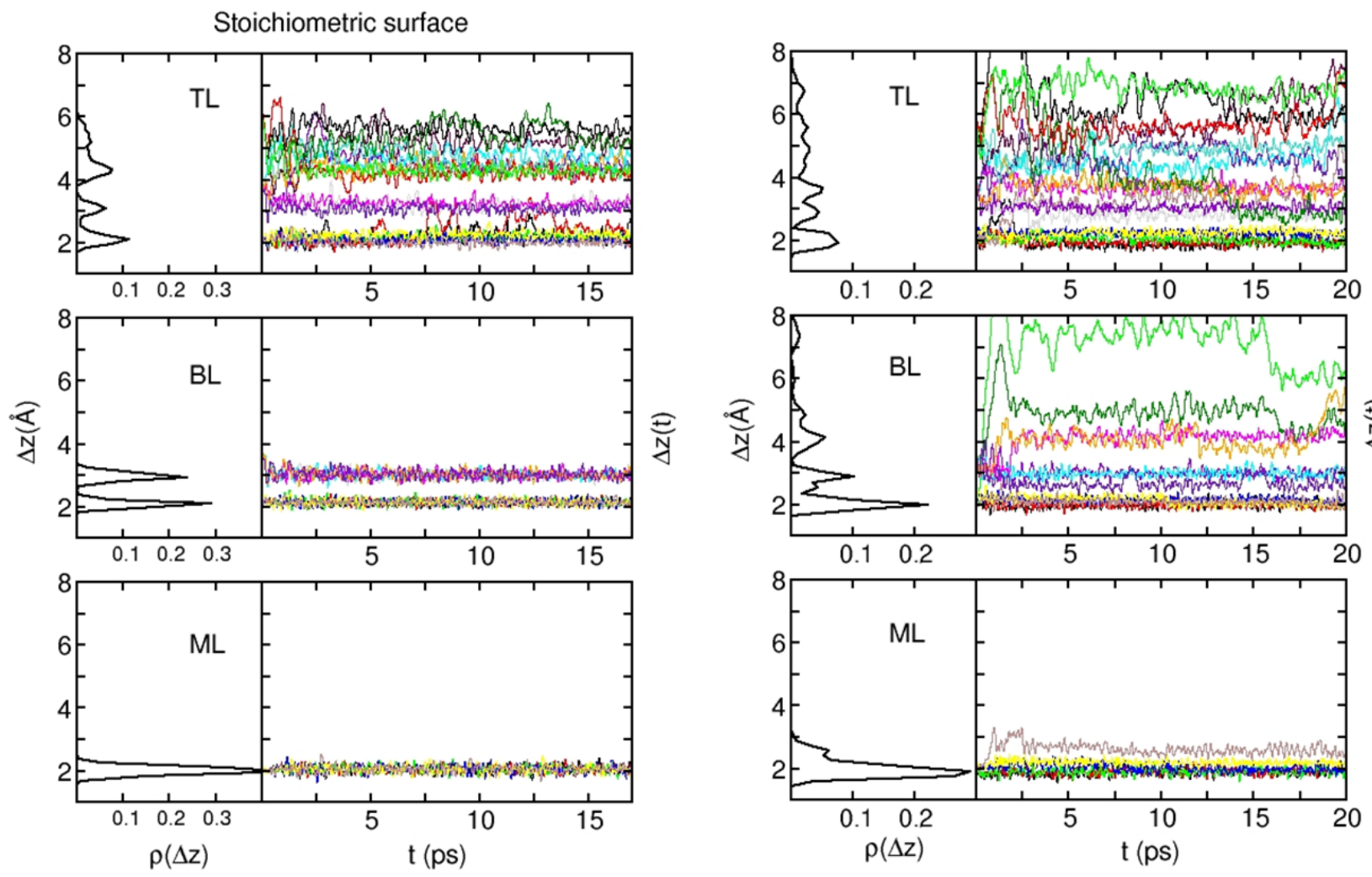

(a)

(b)

Figure 3. Time evolution of the water-surface vertical distances and corresponding probability distributions for adsorbed mono-, bi-, and trilayers of water on the stoichiometric (a) and reduced (b) anatase (101) surface $. \Delta z=z-\bar{z}$, where $\bar{z}$ is the average $\mathrm{z}$ coordinate of the surface $\mathrm{Ti}_{5 \mathrm{c}}$ cations. 

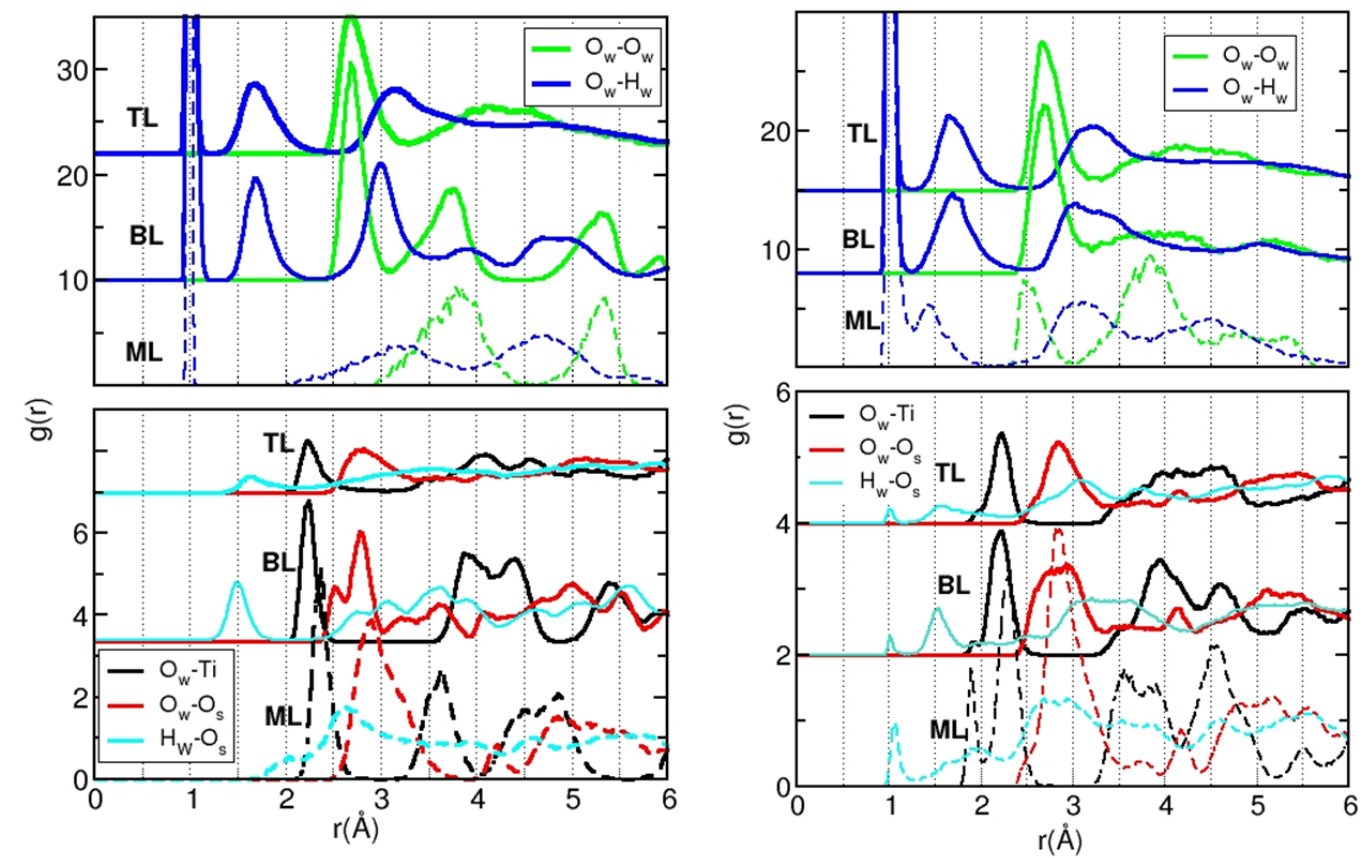

(a)

(b)

Figure 4. Water-water (top) and water-surface (bottom) pair correlation functions for mono-, bi-, and tri-layers of water adsorbed on stoichiometric (a) and reduced (b) anatase (101). The subscripts ' $w$ ' and ' $\mathrm{s}$ ' indicate a water and surface species, respectively. 


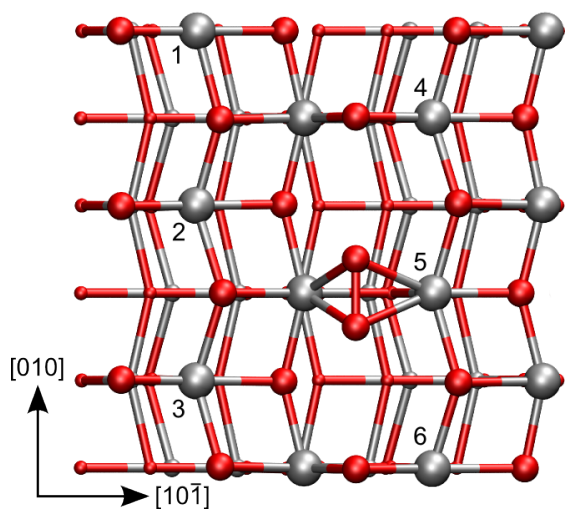

Figure 5: Inequivalent $\mathrm{Ti}_{5 \mathrm{c}}$ adsorption sites on an oxidized surface with a bridging dimer. Atoms in the surface layer are shown as spheres, for lower layers only bonds are shown. Color code: $\mathrm{Ti}=$ silver, $\mathrm{O}=$ red. 

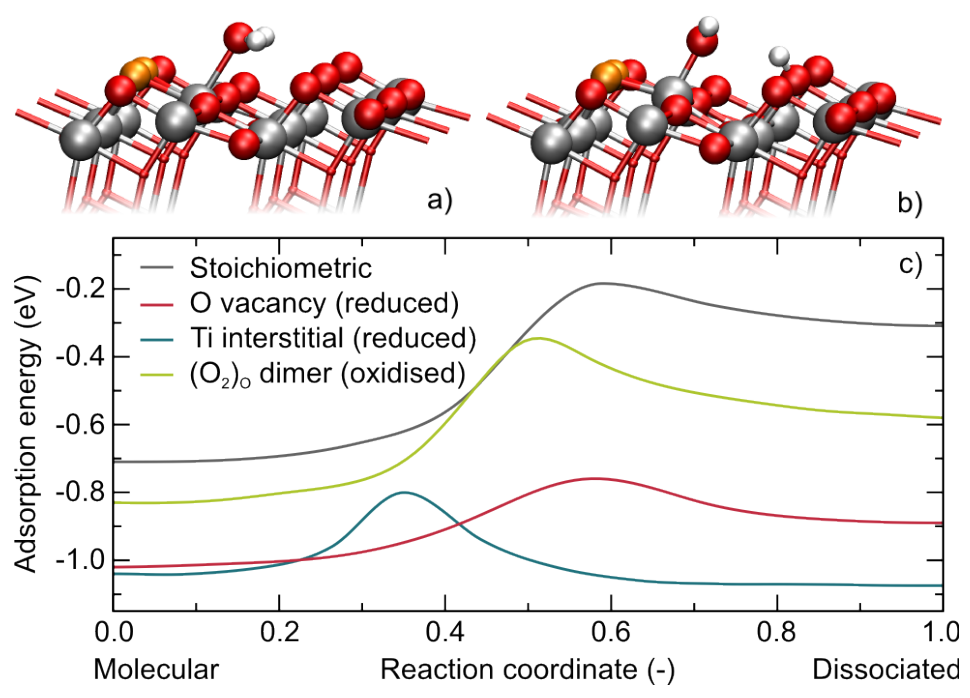

Figure 6 - Upper panel: molecular (a) and dissociated configurations of a water molecule adsorbed on the oxidized surface. The bridging oxygen dimer defect is highlighted in orange. Lower panel: adsorption energy profiles for water dissociation on the stoichiometric, reduced (via either an oxygen vacancy or a Ti interstitial) and oxidized (via the $\left(\mathrm{O}_{2}\right)_{\mathrm{O}}$ dimer) anatase (101) surfaces, computed using the NEB method. The reaction coordinate is defined in such a way that reaction coordinate $=0$ and 1 corresponds to the molecular and dissociated state, respectively. 


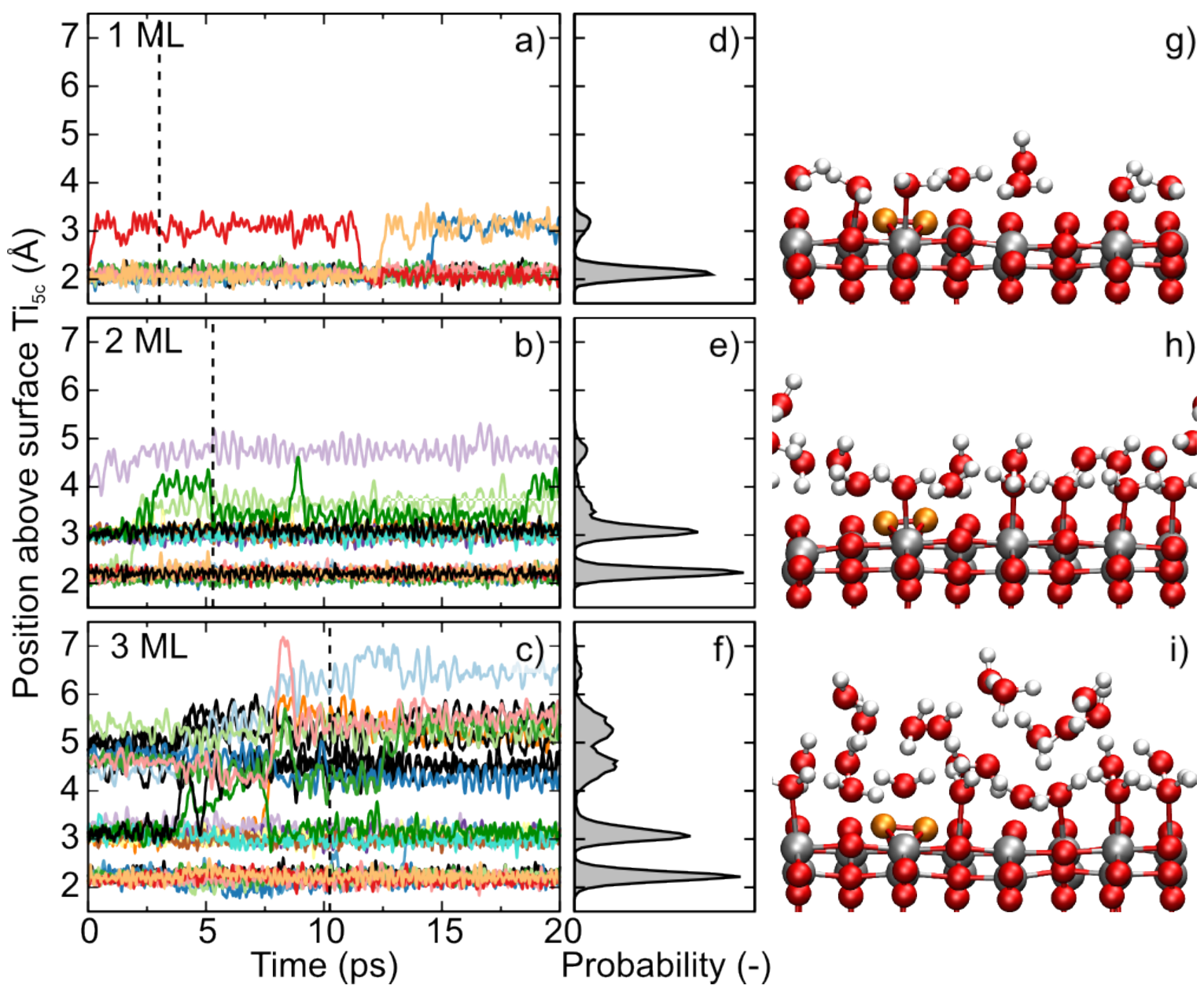

Figure 7: Time evolution of the water-surface vertical distances and corresponding probability distributions for a water monolayer (a, d), bilayer (b, e), and trilayer (c, f). Snapshots for the monolayer $(\mathrm{g})$, bilayer (h), and trilayer (i), viewed along the [-101] direction, with the dimer highlighted in orange; the instant of the snapshot is indicated by the dashed vertical line in a)-c) respectively. 

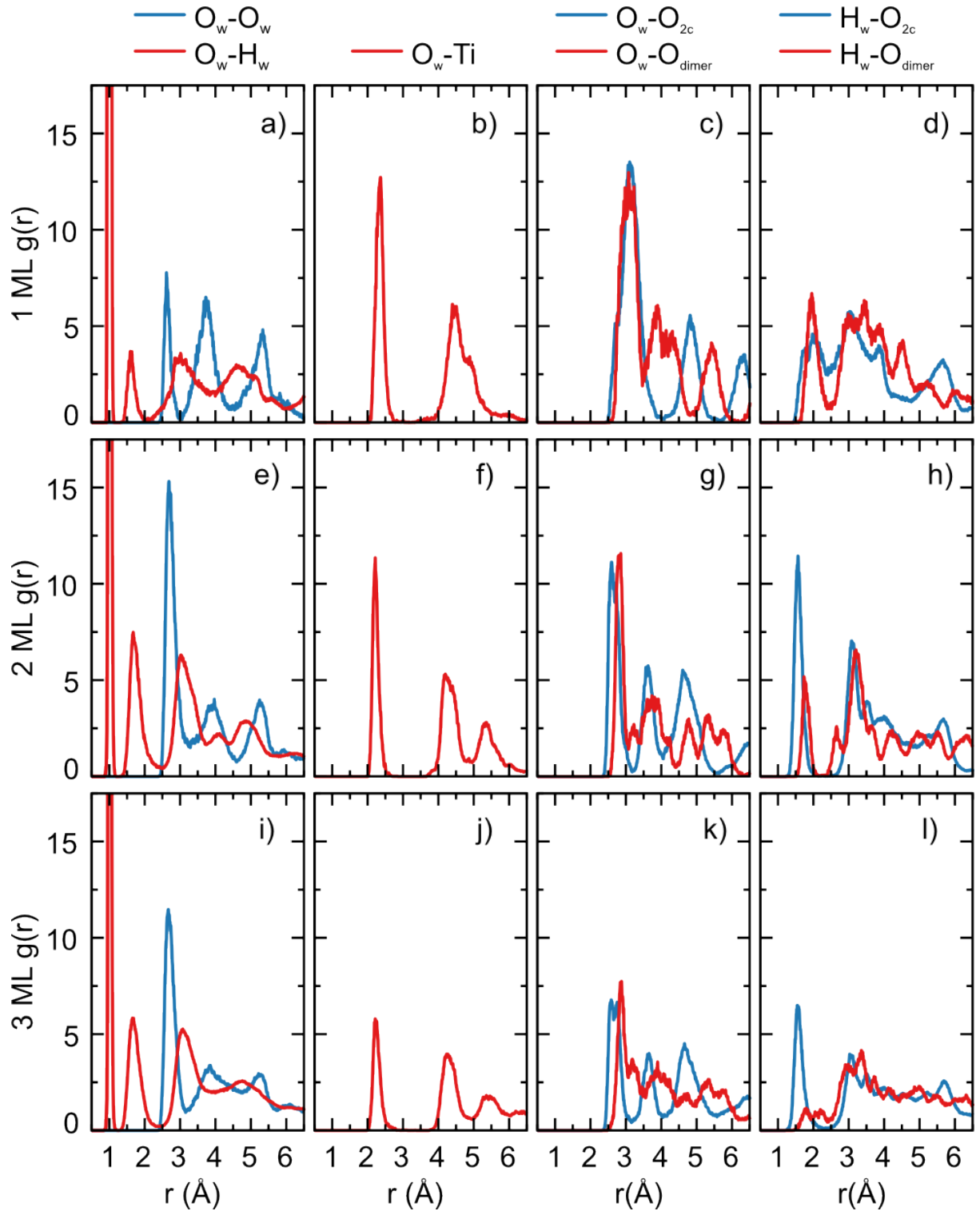

Figure 8: Pair correlation functions for one water monolayer (a-d), bilayer (e-h) and trilayer (i-1). Waterwater pair correlation functions are shown in the first column, while $\mathrm{O}_{\mathrm{w}}-\mathrm{Ti}, \mathrm{O}_{\mathrm{w}}-\mathrm{O}_{\mathrm{s}}$ and $\mathrm{H}_{\mathrm{w}}-\mathrm{O}_{\mathrm{s}}$ correlations are shown in the second, third and fourth column, respectively . 